Eri Kubo - Dhirendra P. Singh • Nigar Fatma •

Toshimichi Shinohara • Peggy Zelenka •

Venkat N. Reddy • Leo T. Chylack

\title{
Cellular distribution of lens epithelium-derived growth factor (LEDGF) in the rat eye: loss of LEDGF from nuclei of differentiating cells
}

\begin{abstract}
Lens epithelium-derived growth factor (LEDGF) enhances the survival and growth of cells. To understand LEDGF's spatial localization and its putative function(s) during proliferation and differentiation, we localized LEDGF during terminal differentiation in whole rat lenses, lens epithelial cell (LEC) explants stimulated with FGF-2, and insulin, iris, human LECs with lentoids. In addition, intracellular localization of LEDGF was performed in other ocular tissues: ciliary body, retina, and cornea. We found the immunopositivity of nuclear LEDGF decreased in LECs of the equatorial region. In contrast, immunopositivity of LEDGF was detected in the cytoplasm of LECs and superficial fiber cells. After treating LEC explants with FGF-2 and insulin, which are known to be differentiating factors for LECs, the nuclei of these cells showed no
\end{abstract}

\author{
D. P. Singh $\cdot$ N. Fatma $\cdot$ T. Shinohara \\ Department of Ophthalmology, Room 2009, \\ University of Nebraska Medical Center, \\ Omaha, NE 68198-6395, USA \\ E. Kubo $\cdot$ D. P. Singh · N. Fatma - T. Shinohara $\cdot$ L. T. Chylack Jr \\ The Center for Ophthalmic Research, \\ Brigham and Women's Hospital, Havard Medical School, \\ 221 Longwood Avenue, Boston, MA 02115, USA \\ E. Kubo \\ Department of Ophthalmology, \\ Fukui Medical University, Fukui, Japan \\ E. Kubo $\cdot$ P. Zelenka \\ Laboratory of Molecular and Developmental Biology, \\ National Eye Institute, National Institutes of Health, \\ Bethesda, MD 20892, USA \\ V. N. Reddy \\ Kellogg Eye Center, \\ Department of Ophthalmology and Visual Science, \\ University of Michigan, Ann Arbor, Michigan, USA \\ D. P. Singh $(\square)$ \\ Wittson Hall, Room 2009, \\ University of Nebraska Medical Center, \\ e-mail: dpsingh@unmc.edu \\ Tel.: +1-402-5598805 \\ Fax: +1-402-5598808
}

LEDGF immunopositivity, but explants did express p5 $7^{\mathrm{kip} 2}$, a differentiation marker protein. Also, immunopositive LEDGF was not detected in the nuclei of differentiated cells, lentoid body, and corneal epithelial cells. This demonstrated that the loss of LEDGF from the nucleus may be associated with the process of terminal differentiation that might be in some way common with the biochemical mechanisms of apoptosis. The spatial and temporal distribution of LEDGF in the present study also provides a vision for further investigation as to how this protein is involved in cell fate determination.

Keywords LEDGF - Immunohistochemistry · Differentiation - In situ hybridization - Lentoid body . LEC explants

\section{Introduction}

The ocular lens contains three principal cell types: central lens epithelial cells (LECs), germinative zone epithelial cells, and fiber cells. In the human lens, most central LECs survive for decades without dividing or differentiating, but approximately $14 \%$ of these cells die over the course of a 75-year lifetime, leaving gaps or areas covered by attenuated extensions of adjacent cells in the central epithelial monolayer (Balaram et al. 2000). The germinative LECs, found in the pre-equatorial region of the lens, are aptly named, for they divide and differentiate into elongated, fusiform, crystallin-rich, organelle-deficient, anucleate fiber cells. This transformation is called terminal differentiation (TD) or fiber differentiation. The TD begins in epithelial cells of the bow region and proceeds to completion in the deeper layers of the superficial cortex (Piatigorski 1981). Many investigations have greatly increased our understanding of the roles of growth factors in TD. For example, acidic and basic fibroblast growth factors (FGF-1 and FGF-2, respectively; Chamberlain and McAvoy 1987, 1989; Lovicu and McAvoy 1989; McAvoy and Chamberlain 1989), insulin growth factor-1 along with FGF (Chamberlain et al. 1991; 
Klok et al. 1998), and epidermal growth factor (Ibaraki et al. 1995) all induce TD in LECs.

In contrast to the abundant research on growth factors and TD in the lens, there are few studies on factors involved in LEC survival and death. In 1992-3, Ishizaki et al. (1993) and Raff (1992) showed that LECs secreted survival factors, but they were not able to identify them. Renaud et al. (1994) showed that endogenous FGF-1 expression was higher in non-dividing LECs than in exponentially dividing cells and suggested that FGF-1 was not a mitogen but a survival factor. Also Stolen et al. (1997) demonstrated that FGF-2 modulated not only the late stages of differentiation, but also the survival of lens fiber cells. We have reported that lens epithelium-derived growth factor (LEDGF) enhances survival, adhesiveness, and growth of mouse LECs and other cell types in culture (Singh et al. 1999, 2000a; Nakamura et al. 2000) and we have shown that heparin even further enhances LEDGF's effects on cell growth and survival (Fatma et al. 2001). Furthermore, LEDGF is a novel transcriptional factor (Fatma et al. 2001; Singh et al. 2001a) and is identical with p75, a known co-activator of transcription (Ge et al. 1998a, b; Singh et al. 2000b). If overexpressed in LECs, LEDGF increased LEC resistance to various stresses (thermal, oxidative) and upregulated the expression of heat shock proteins, Hsp27, antioxidant protein 2, and $\alpha \mathrm{B}$-crystallin by binding to specific sites in each gene promoter (Singh et al. 1999, 2001a; Fatma et al. 2001). LEDGF was also upregulated when cells were exposed to thermal and oxidative stresses (Sharma et al. 2000). We have shown that the upregulation of stress proteins by LEDGF is one of the mechanisms by which LEDGF prolongs LEC survival (Singh et al. 1999).

This paper describes the spatial distribution of LEDGF protein and LEDGF mRNA in tissues of the rat eye using immunohistochemical and in situ hybridization techniques. We have also studied the effect of age on this distribution. The findings described herein link changes in the immunoreactivity of nuclear LEDGF levels to TD in four model systems: whole rat lens, rat LEC explants in which differentiation has been induced, lentoids produced in cultured human LECs, and cornea. The immunoreactivity of $\mathrm{p} 57^{\mathrm{kip} 2}$, a differentiation marker, was also examined in rat LEC explants.

\section{Materials and methods}

\section{Animals}

We used 3-, 7-, and 13-week-old Sprague-Dawley albino rats (Charles River, Wilmington, Mass., USA). All animals were handled in accordance with the ARVO Policies on the Use of Animals in Research. The rats were euthanized with $\mathrm{CO}_{2}$ and then perfused through the left ventricle with $4 \%$ paraformaldehyde (PFA) in $20 \mathrm{mM}$ phosphate-buffered saline (PBS), pH 7.4.
Antibody generation, production, purification, and neutralization

A polyclonal antibody $(\mathrm{Ab})$ to a C-terminal peptide of human LEDGF (YNKFKNMFLVGEGDSVITQ) corresponding to the amino acid sequence (420-438) (Singh et al. 2001a) was raised in rabbits. This C-terminal region does not share homology with any known protein as determined by computer analysis program, BLASTp, the Basic Local Alignment Search Tool for Protein developed by NCBI, National Center for Biotechnology Information. The IgG fraction of the immune serum was affinity purified with Immunopure Immobilized Protein A (Pierce, Rockford, Ill., USA) column chromatography and concentrated with Centricon Plus 20 (Millipore, Bedford, Mass., USA). We neutralized the purified anti-LEDGF $\mathrm{Ab}$ by adding $250 \mu \mathrm{l}$ keyhole limpet hemocyanin (KLH) and $500 \mu \mathrm{g}$ GST-LEDGF to a 500- $\mu$ l aliquot of purified $\mathrm{Ab}$ preparation. The techniques for producing and purifying the GST-LEDGF fusion protein have been published (Fatma et al. 2000). We controlled for non-specific Ab absorption by adding $250 \mu \mathrm{l} \mathrm{KLH}$ and $500 \mu \mathrm{g}$ bovine serum albumin (BSA) to a second $500 \mu \mathrm{l}$ aliquot of the purified Ab preparation, and both solutions were incubated overnight at $4{ }^{\circ} \mathrm{C}$ and then centrifuged at $8,000 \mathrm{rpm}$ for $5 \mathrm{~min}$. The supernatants of these two solutions were designated the "GST-LEDGF-neutralized Ab" and the "anti-(Cterminal) LEDGF Ab" preparations. The concentration of protein in each Ab preparation was measured by the Bradford (1976) method and equalized at $1.28 \mathrm{mg} / \mathrm{ml}$. P57 $7^{\mathrm{kip} 2}$ antibody (Santa Cruz Biotechnology, Santa Cruz, Calif., USA) was used as differentiation marker.

\section{Preparation of tissue sections}

The lens was fixed for $48 \mathrm{~h}$ in $4 \%$ PFA in PBS, and other ocular tissues were dissected and immersed in the same solution for $24 \mathrm{~h}$. Cornea, lens, iris, ciliary body, and retina were embedded in paraffin (Paraplast Plus; Oxford Labware, St. Louis, Mo., USA) and sectioned at $4 \mu \mathrm{m}$. The sections were deparaffinized in xylene and immersed in a graded series of ethanol and xylene. For cryosectioning, lenses were fixed for $48 \mathrm{~h}$ in $4 \%$ PFA in PBS and embedded in OCT compound (Tissue-Tek; Sakura Finetek, Torrance, Calif., USA), frozen, and then sectioned on a cryostat at $10 \mu \mathrm{m}$. The frozen sections were dried, washed in PBS, and then immersed in cold $100 \%$ methanol at $-20^{\circ} \mathrm{C}$ for $2 \mathrm{~min}$.

Flat preparations of rat lens epithelial cells

Lenses from 3-week-old rats were placed on a polyvinylidene fluoride (PVDF) membrane (Immobilon-P; Millipore). The posterior lens capsule was opened and the lens fibers were removed from the epithelial layer and capsule en masse. The remaining capsule epithelium was spread on the PVDF membrane, and the membrane

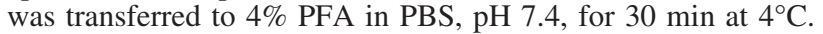

Preparation of paraffin sections of cultured human lentoids

Paraffin blocks containing human LECs (HLECs) and lentoid bodies were prepared as follows. The initial monolayer of cells obtained from anterior capsule explants after 2 weeks were trypsinized and subcultured twice to obtain HLECs as described previously (Arita et al. 1988, 1990). After the second subculture (passage 2) $1 \times 10^{5}$ cells were plated on Millipore CM 10-mm inserts (Millipore) in 12-well plates. The HLECs were cultured in DMEM medium (Gibco BRL, Grand Island, N.Y., USA) containing 20\% fetal bovine serum. The medium was changed twice each week. By the 4th day, lentoids had formed on the surface of the membrane. The lentoids were fixed with 3\% PFA in PBS after 2 and 3 weeks of incubation, embedded in paraffin, and sectioned at approximately $4 \mu \mathrm{m}$. 
Preparation of rat LEC explants

Lens epithelial explants were cultured, and paraffin sections were prepared as previously described (Rampalli and Zelenka 1995). Briefly, lenses were removed from 4-day-old rats, and the lens epithelium was separated from the fiber mass through a tear in the posterior capsule. A square explant was cut from the central region of the lens epithelium and pinned down to the surface of the culture dish with the capsular surface toward the plastic. Differentiation was initiated by adding $1 \mu \mathrm{g} / \mathrm{ml}$ porcine insulin (Gibco) and $100 \mathrm{ng} /$ $\mathrm{ml}$ human FGF-2 (Gibco) to Medium 199 (Gibco) containing penicillin and streptomycin and incubating the cells for 6,24 , and $48 \mathrm{~h}$. Control explants $(0 \mathrm{~h})$ were fixed immediately without culturing. All epithelia were fixed in $4 \%$ PFA in PBS at room temperature for $2-3 \mathrm{~h}$. The epithelia were embedded in paraffin and sectioned at $7 \mu \mathrm{m}$ thickness.

Immunohistochemical staining

After washing in PBS, all sections were placed in $0.3 \%$ hydrogen peroxide in methanol for 30 min to block endogenous peroxidase activity. Paraffin sections from rat eye tissues and flat preparation of rat lens were immersed in $0.1 \%$ trypsin for $25 \mathrm{~min}$, and paraffin sections of cultured LECs and explants were immersed in $0.1 \%$ trypsin for 2 min. After washing in PBS, all tissue sections and flat preparations were incubated for $30 \mathrm{~min}$ with $10 \%$ normal goat serum (Dako, Carpinteria, Calif., USA). Then they were exposed overnight at $4{ }^{\circ} \mathrm{C}$ to the anti-(C-terminal) LEDGF $\mathrm{Ab}$ in PBS containing $5 \%$ dry milk or $10 \%$ goat serum. Antibody preparations were diluted 1:500 for use with frozen lens sections, and 1:750 for use with paraffin sections and flat preparations. After washing in PBS, these tissue sections were exposed to biotinylated anti-rabbit immunoglobulins in PBS containing carrier protein (Dako) for $2 \mathrm{~h}$ at room temperature and then washed again in PBS. The specimens were incubated in avidin-peroxidase-conjugated streptavidin (Dako) at room temperature for $2 \mathrm{~h}$. Visualization of the antibody complex was carried out by adding a $0.02 \%$ solution of $3,3^{\prime}$ diaminobenzidine (DAB; BioRad Laboratories, Hercules, Calif., USA) and $0.05 \%$ hydrogen peroxide in TRIS-buffered saline, $\mathrm{pH}$ 7.6, containing $1 \mathrm{mM} \mathrm{CaCl}$. Negative controls were incubated with the GST-LEDGF-neutralized Ab preparation overnight at $4^{\circ} \mathrm{C}$. All sections, except the frozen sections and explants, were counterstained with Mayer's hematoxylin (Sigma, St. Louis, Mo., USA). In rat LEC explants, sections for fluorescence probes were also immunostained with anti-LEDGF Ab diluted 1:1,000 with 10\% goat serum and anti-goat p57 $7^{\text {kip2 }}$ Ab (Santa Cruz Biotechnology) diluted 1:200 with 5\% BSA in PBS. As secondary Abs we used anti-rabbit IgG-HRP or anti-goat IgG-HRP (Santa Cruz Biotechnology). Sections were visualized with cyanin-3 tyramide fluorescence probe (NEN Life Science, Boston, Mass., USA).

\section{Protein blot analysis}

Lens capsule epithelium and whole retina were obtained from each of fifteen 3-week-old rats. The capsule epithelia and retinas were pooled and suspended in 1.0-ml aliquots of ice-cold RIPA buffer (1\% Igepal CA-630; Sigma), $0.5 \%$ sodium deoxycholate, $0.1 \%$ sodium dodecyl sulfate (SDS; Gibco) containing 1,000 U/ml heparin (Gibco), $1 \mathrm{mM}$ phenylmethylsulfonyl fluoride (Sigma), and one protease inhibitor cocktail tablet (Complete; Roche, Mannheim, Germany) per $50 \mathrm{ml}$ to protect LEDGF from degradation (Fatma et al. 2000). Tissue specimens were homogenized with a Dounce homogenizer, centrifuged at $12,000 \mathrm{rpm}$ for $15 \mathrm{~min}$ at $4{ }^{\circ} \mathrm{C}$, and the supernatant was collected. The protein concentration of each supernatant was determined by the Bradford (1976) method. The protein lysate was mixed with SDS polyacrylamide gel electrophoresis (SDS-PAGE) sample buffer $(1: 1, \mathrm{v} / \mathrm{v})$, and samples were run on a $7.5 \%$ SDS-PAGE gel, transferred to a PVDF membrane (Immobilon-P; Millipore), and blocked with PBS-T (PBS containing $0.05 \%$ Tween 20 ), $7 \%$ dry milk, and $10 \%$ goat serum. The blots were incubated overnight at $4{ }^{\circ} \mathrm{C}$ with affinitypurified rabbit anti-(C-terminal) LEDGF IgG (diluted 1:2,000 in PBS-T containing 5\% dry milk and 5\% goat serum. After washing in PBS-T, the membranes were incubated with goat anti-rabbit IgG labeled with horseradish peroxidase (diluted 1:2,000; Santa Cruz Biotechnology) for $2 \mathrm{~h}$ at room temperature and visualized by enhanced chemiluminescence according to the company's protocol (Western Blotting Luminol Reagent; Santa Cruz Biotechnology). Negative controls were incubated overnight with the GST-LEDGFneutralized $\mathrm{Ab}$ preparation at $4^{\circ} \mathrm{C}$. Full-size GST-LEDGF (MW $89 \mathrm{kDa}$ ) was run with these samples as a positive control.

\section{In situ hybridization}

After killing the 3-week-old rats, the eyes were rapidly removed and fixed with $4 \%$ PFA in $0.1 \%$ diethyl pyrocarbonate-treated PBS Lenses were embedded in paraffin and sectioned at $4 \mu \mathrm{m}$. The following 48-bp antisense LEDGF oligonucleotide probe was used: 5'-GCT CTC CCC GTT ATG TTG TGG CTG ATT ACC ATC TTG AGC ATC AGA TCC-3' (Life Technologies, Rockville, Md., USA). The GC content of this probe was $50 \%$. This sequence corresponded to nucleotides 1751-1798 of the LEDGF cDNA sequence (Singh et al. 2000b), which is specific for LEDGF and would not pick up p52-specific transcripts. Control hybridizations were conducted using a corresponding 48-bp sense probe. An antisense mouse GAPDH RNA probe provided by the company (Ambion, Austin, Tex., USA) was used as a positive control. All probes were labeled with biotin using a Psoralen Biotin conjugate according to the protocol in the Ambion's BrightStar PsoralenBiotin Nonisotopic Labeling kit. Briefly, the probes were diluted in TE (10 mM TRIS-HCl, pH 8, $1 \mathrm{mM}$ EDTA) at a concentration of $50 \mathrm{ng} / \mu \mathrm{l}$ and then transferred to a well in the microtiter plate. Psoralen-Biotin reagent, $1 \mu \mathrm{g} / 10 \mu \mathrm{l}$, was added to each well containing the probes. A 365 -nm UV light source was placed on the plate directly over the samples, and the samples were irradiated for $45 \mathrm{~min}$. Non-crosslinked Psoralen-Biotin reagent was removed with distilled $\mathrm{H}_{2} \mathrm{O}$ saturated with n-butanol. The following in situ hybridization procedure was performed using Ambion's mRNAlocator-Hyb kit. The labeled antisense and sense probes were diluted to a final concentration of $10 \mathrm{ng} / 100 \mu \mathrm{l}$ (per each slide) with kit hybridization buffer. The labeled GAPDH probes were diluted to a final concentration of $1 \mathrm{ng} / 100 \mu \mathrm{l}$ (per slide) with hybridization buffer. Sections were hybridized with the labeled probes at $65^{\circ} \mathrm{C}$ for $5 \mathrm{~min}$ followed by hybridization at $42^{\circ} \mathrm{C}$ overnight (for antisense and sense LEDGF probes) and at $50^{\circ} \mathrm{C}$ overnight (for the GAPDH probe). The slides were then washed with the kit's in situ wash buffer at $55^{\circ} \mathrm{C}$ for $30 \mathrm{~min}$. Biotin detection procedure was performed using Ambion's mRNAlocator-Biotin kit. Briefly, sections were incubated with streptavidin-alkaline phosphatase conjugate (1:300 dilution with $1 \times$ TRIS buffer) at $37^{\circ} \mathrm{C}$ for $1 \mathrm{~h}$. After washing in $1 \times$ TRIS buffer, sections were incubated with nitro blue tetrazolium salt/5-bromo-4-chloro-3-indolyl phosphate in bimethylformamide solution at $37^{\circ} \mathrm{C}$ for $1 \mathrm{~h}$. We monitored the color development, then dehydrated and mounted the sections. Photomicrographs were taken with brightfield microscopy.

\section{Results}

\section{Protein blot analysis of LEDGF in rat lens and retina}

We first monitored the specificity of anti-LEDGF antibody to GST-LEDGF recombinant protein, and the presence of LEDGF in LECs and retina using protein blot analysis. Figure 1 presents western blots of purified GSTLEDGF (Fig. 1 lanes $A, B$ ), LEDGF from rat lens capsule epithelium (Fig. 1 lanes $C, D$ ), and rat retina (Fig. 1 lanes $E, F)$ using the anti-(C-terminal $) \mathrm{Ab}$ preparation 


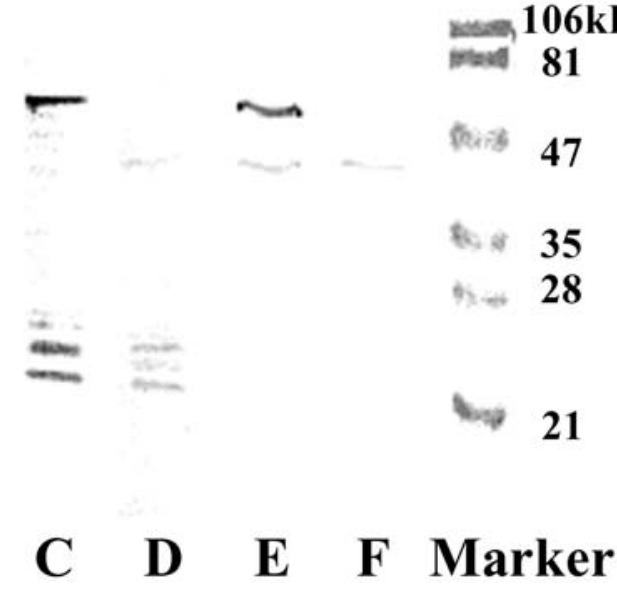

Fig. 1 Protein blot analysis of lens epithelial cell (LEC) and retinal proteins with anti-lens epithelium-derived growth factor (LEDGF) antibody. Purified GST-LEDGF (lanes A, B), total rat LEC preparation (lanes $C, D$ ), and rat retinal cell preparation (lanes $E, F$ ) were immunostained with anti-LEDGF antibody $(\mathrm{Ab}$; lanes $A, C, E)$ and with the GST-LEDGF-neutralized LEDGF $\mathrm{Ab}$ (lanes $B, D, F)$. Stained protein bands at 89 and $60 \mathrm{kDa}$ denote GST-LEDGF and native LEDGF, respectively. Marker lane contains molecular weight markers in kilodaltons

(Fig. 1 lanes $A, C, E$ ) and the GST-LEDGF-neutralized $\mathrm{Ab}$ preparation (Fig. 1 lanes $B, D, F$ ). In Fig. 1 lane $A$ note the strong staining at $89 \mathrm{kDa}$, the MW of the GSTLEDGF conjugate (GST MW=29 kDa; LEDGF $\mathrm{MW}=60 \mathrm{kDa})$. In Fig. 1 lane $C$, the strong band at $60 \mathrm{kDa}$ (endogenous LEDGF) and some minor bands were observed. Figure 1 lanes $B, D, F$ shows the disappearance of the 89 and $60 \mathrm{kDa}$ bands. Some of the minor bands at approximately 28 and $50 \mathrm{kDa}$ (capsule epithelium) and $43 \mathrm{kDa}$ (retina) that stain non-specifically did not disappear in the absorbed Ab preparation.

Immunohistological localization of LEDGF in rat lens

\section{Cross-sections}

The immunohistological localization of LEDGF in the 3week-old rat lens is presented in Fig. 2 (paraffin section). The nuclei and cytoplasm of LECs in the germinative zones (Fig. 2A, B) were stained strongly. The nuclei of LECs in the central epithelium were also stained (data not shown). As one approaches the equator, the intensity of nuclear staining diminishes markedly (Fig. 2B), and at or near the postequator, the nuclei do not stain. The cytoplasm of the superficial fibers in the anterior cortex is only weakly immunopositive for LEDGF while the cytoplasm of the superficial fibers in the posterior cortex is strongly immunopositive (Fig. 2A, B). The intense staining of cytoplasmic LEDGF evident in the most superficial equatorial and posterior lens fibers, appears diminished in the deeper cortical fibers (Fig. 2A). The intensity of immunostaining was significantly reduced in the sections stained with the GST-LEDGF-neutralized $\mathrm{Ab}$ (Fig. 2C). The pattern of LEDGF immunostaining of the lens did not vary with age among rats at 3,9, and 13 weeks of age (data for 9- and 13-week-old rats not shown). These results indicate that the pattern of expression of LEDGF varies with the region in the lens. We chose to confirm this by looking at LEDGF expression in flat preparations of lens epithelium.

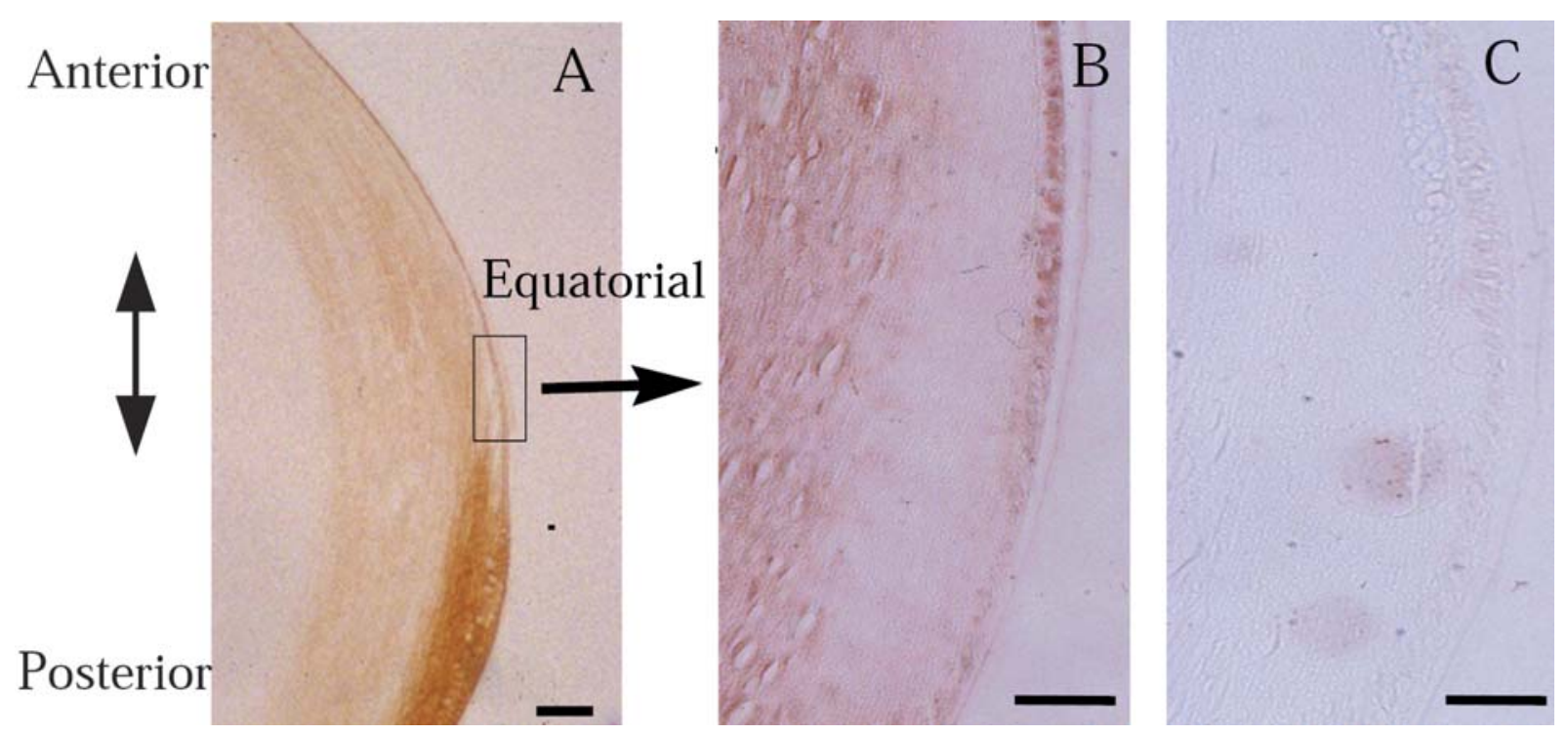

Fig. 2A-C Immunohistochemical staining of LEDGF in the 3week-old rat lens. Paraffin sections of rat lens immunostained with anti-LEDGF $\mathrm{Ab}(\mathbf{A})$ or with LEDGF-neutralized $\mathrm{Ab}(\mathbf{B}, \mathbf{C})$. The intense immunostaining of nuclei, the weak staining of the cytoplasm in the germinative LECs, and the gradual loss of staining in the nuclei of the equatorial bow region and the nucleated superficial fiber cells were observed. Cortical fibers in the posterior region were strongly immunostained with anti-LEDGF Ab. A Bar $120 \mu \mathrm{m}$, magnification $\times 40$. B, C Bar $80 \mu \mathrm{m}$, magnification $\times 100$ 


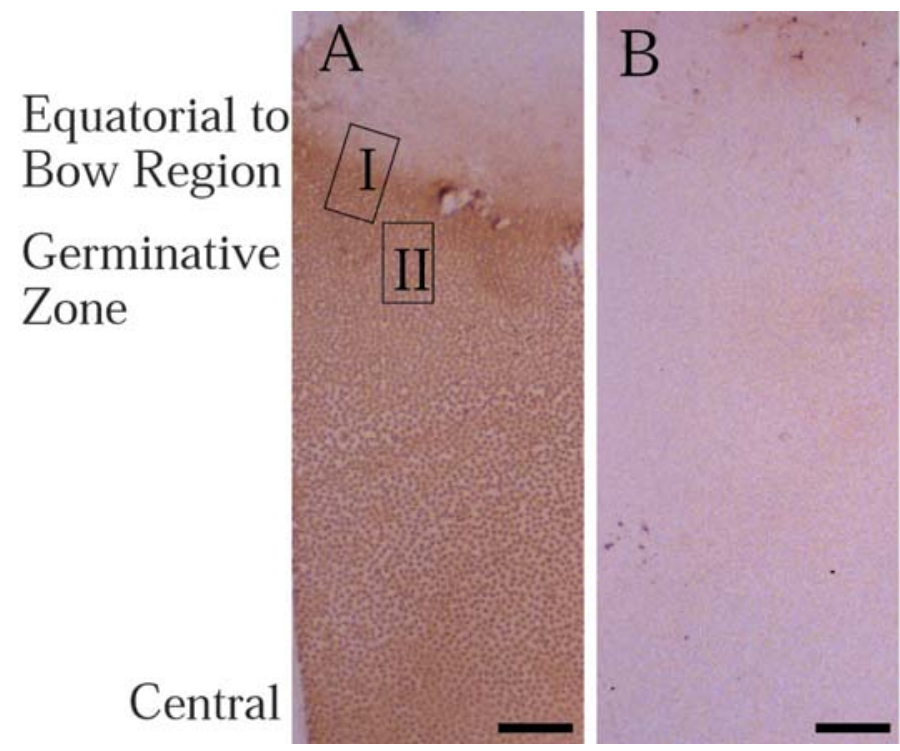

Fig. 3A, B Immunohistochemical staining of LEDGF and counterstaining with hematoxylin of flat preparations of the lens epithelium from a 3-week-old rat. The capsule epithelium stained with anti-LEDGF $\mathrm{Ab}(\mathbf{A})$. Insets $I$ and $I I$ indicate the germinative zones $(I)$ and anterior LECs $(I I)$. Anterior LECs showed intense

\section{Flat preparations}

The diminished immunoreactivity of nuclear LEDGF in cells of the equatorial bow regions, evident in crosssections of whole lens (Fig. 2B), was even more apparent in flat preparations of LECs from 3-week-old rats (Fig. 3 insets AI, AII). The nuclei in the central epithelium, and in the sample from the germinative zone, all showed strong immunostaining with anti-LEDGF $\mathrm{Ab}$. In the germinative zone, nuclear LEDGF immunostaining was significantly diminished (Fig. 3 inset AI). All magnified views of these zones (Fig. 3 insets AI, AII) were derived from the same flat preparation, and they demonstrate the rank order of nuclear staining intensity: central region $>$ germinative region $>$ bow region.

Furthermore, the staining of the cytoplasm in all these regions was generally much less intense and more varied than in the nuclei. In control sections stained with the GST-LEDGF-neutralized Ab preparation, LEDGF immunostaining was significantly reduced (Fig. 3B); this confirms the lack of non-specific staining and the specificity of the signal derived with the anti-(C-terminal) $\mathrm{Ab}$ preparation. These findings suggest that the loss of nuclear LEDGF in differentiating LECs may reflect the change in functional status of the cell.

Nuclear LEDGF was undetected in lentoid body and explants of cells

We extended our study to include two well-defined fiber cell differentiation systems: (1) lentoid bodies comprised of aggregated LECs grown on agar-coated plates (Arita et
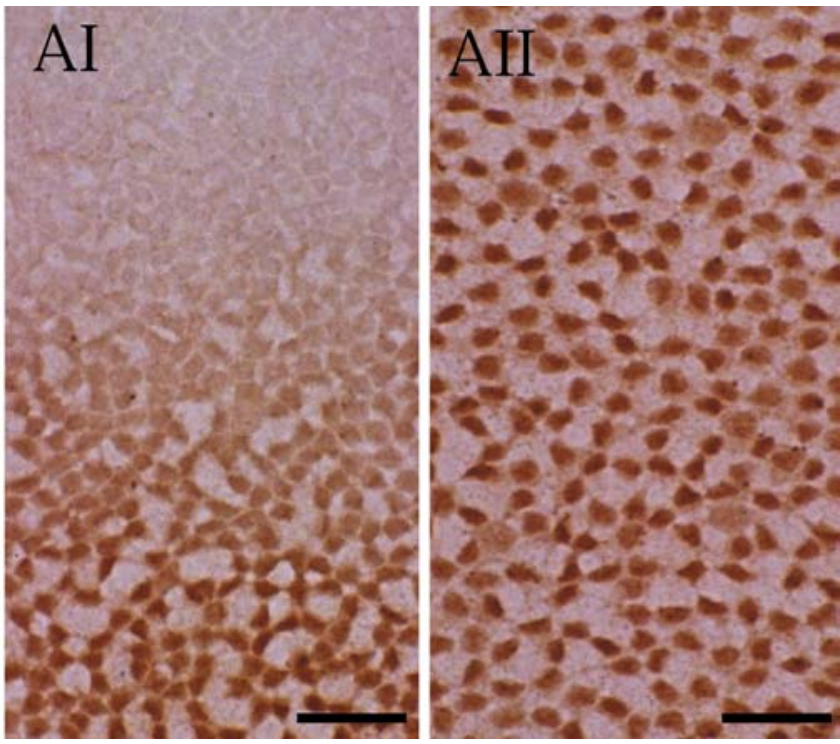

immunostaining in nucleus. A gradual loss of nuclear LEDGF in cells of the equatorial bow region $(I)$ is evident. In control sections stained with neutralized LEDGF Ab, LEDGF staining is significantly reduced (B). A, B Bar $160 \mu \mathrm{m}$, magnification $\times 25$. AI, AII Bar $100 \mu \mathrm{m}$, magnification $\times 150$
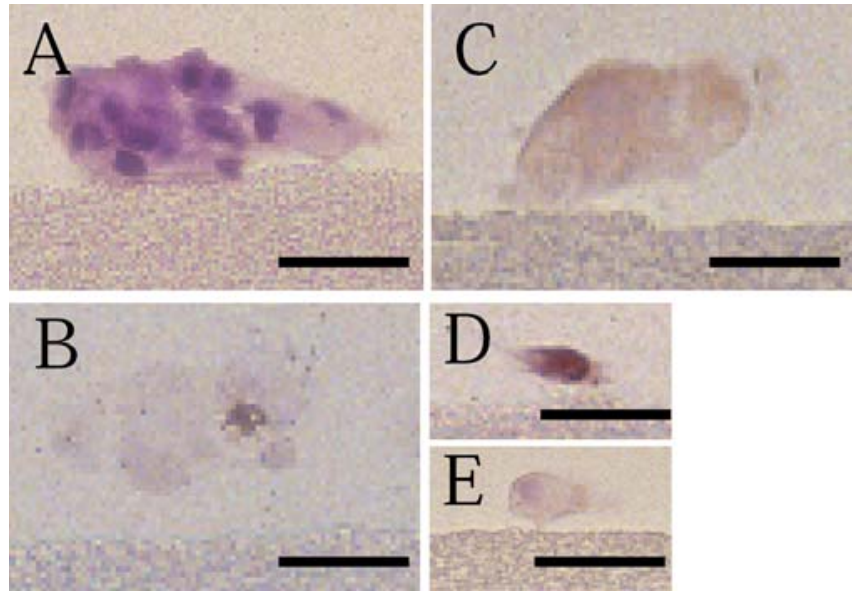

Fig. 4A-E Immunohistochemical staining and counterstaining with hematoxylin and eosin-stained lentoid bodies and single LEC. Thin paraffin sections of the lentoid body were stained with hematoxylin and eosin (A), with anti-LEDGF Ab (B), and with neutralized LEDGF Ab (C). Single LEC stained with anti-LEDGF $\mathrm{Ab}$ (D) or with LEDGF-neutralized Ab (E). A-E Bar $20 \mu \mathrm{m}$, magnification $\times 150$

al. 1988, 1990) and (2) LEC explants exposed to differentiating factors (Rampalli and Zelenka 1995). LECs were cultured for 2-3 weeks on Millipore CM inserts (Millipore) to generate lentoid bodies (Fig. 4A-C). Single LECs were also cultured in 96-well plates as a control vehicle (Fig. 4D, E). Subsequently, they were subjected to immunostaining with anti-LEDGF $\mathrm{Ab}$ to localize LEDGF. Prior to immunostaining, the sections of lentoid body were stained with hematoxylin and eosin 


\section{Antibodies Anti-LEDGF Ab}

\section{Treatment}

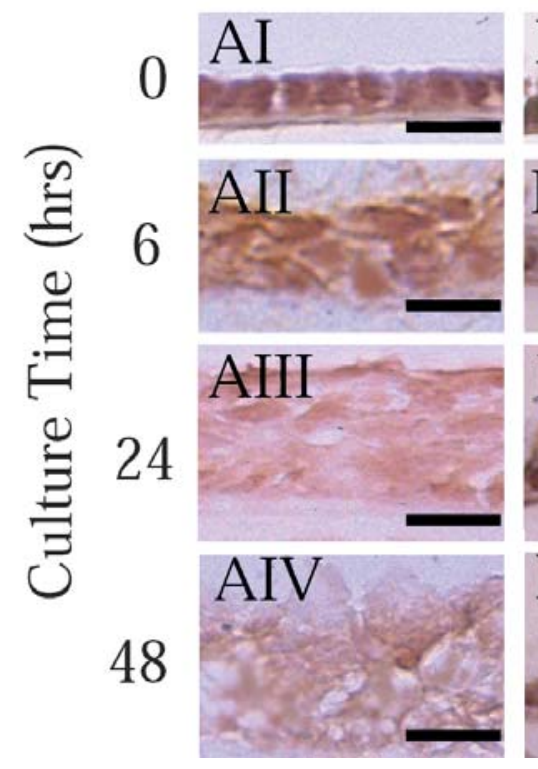

Fig. 5A-D Immunohistochemical staining of LEDGF and p57 $7^{\mathrm{kip} 2}$ in rat LEC explants. The explants were fixed with $4 \%$ paraformaldehyde in phosphate-buffered saline either after $0(I), 6(I I), 24$ (III), and 48 (IV) h exposure to basic fibroblast growth factor (FGF2) and insulin or no exposure. The explants were stained with the anti-LEDGF antibody (A-C) or the anti-p57 ${ }^{\text {kip2 }} \mathrm{Ab}$ (D). The explants were visualized with DAB-colorimetric assay (showing brown color; $\mathbf{A} I-I V, \mathbf{B} I-I V)$ and counterstained with hematoxylin

which showed the presence of nuclei (Fig. 4A). In the section of lentoid bodies, immunostaining of LEDGF in the cell's nucleus was almost negative, where as it was positive in cytoplasm (Fig. 4B); by contrast, immunostaining of a single LEC with anti-LEDGF Ab, showed strong immunopositivity for LEDGF in nucleus and weak in the cytoplasm (Fig. 4D). In control sections immunostained with the neutralized Ab, LEDGF immunostaining was significantly diminished in the lentoid bodies as well as in the single LEC (Fig. 4C, E).

We used differentiation factors to differentiate LECs into lens fiber cells, by culturing explants of LECs in presence of differentiation factors (Rampalli and Zelenka 1995). Rat LEC explants were cultured immediately after removing the cortex and nucleus through a posterior opening in the lens and treated with or without FGF-2 and insulin for 6, 24, and $48 \mathrm{~h}$. Thereafter, the samples were fixed with $4 \%$ PFA in PBS and thin sections were prepared and immunostained either with anti-LEDGF $\mathrm{Ab}$ or LEDGF-neutralized $\mathrm{Ab}$ or anti-p57 ${ }^{\mathrm{kip} 2} \mathrm{Ab}$ (Fig. 5). The explants in Fig. 5AI-IV and $\mathrm{B} I-I V$ have been visualized with $\mathrm{DAB}$ and were slightly stained with hematoxylin, and those in Fig. 5CI-IV and DI-IV were visualized with cyanin-3 fluorescence probes. With both staining systems, DAB and cyanin-3, LEDGF immunostaining was similar (compare Fig. 5AI-IV and $\mathrm{C} I-I V$ ). Furthermore, we

\section{Anti-LEDGF Anti-p57kip2}

\section{FGF+Insulin}
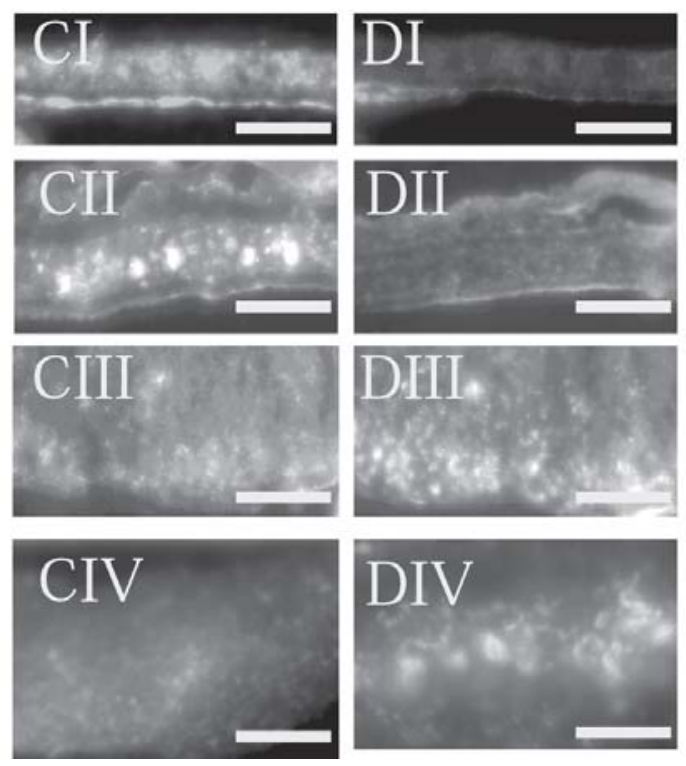

$(\mathbf{A} I-I V, \mathbf{B} I-I V)$. Sections were also visualized with cyanin-3 fluorescence probes (showing with white color; $\mathbf{C} I-I V, \mathbf{D} I-I V$ ). Cells exposed to FGF-2 and insulin differentiated into elongated fibers-like cells $(I I I, I V)$, and untreated cells were single layered (B). The section stained with neutralized LEDGF Ab (photograph not shown) and with anti $557^{\mathrm{kip} 2} \mathrm{Ab}(\mathbf{D} I-I V)$ showed significant reduced immunostaining. Bars $20 \mu \mathrm{m}$, magnification $\times 150$

observed that the explanted LECs that had been exposed to FGF-2 and insulin had elongated, but there was no distinguishable change in untreated explants (Fig. 5BI-IV; Rampalli and Zelenka 1995). In explanted LECs cultured without or with $6 \mathrm{~h}$ exposure to FGF and insulin, positive LEDGF immunostaining was observed predominately in the nucleus and weakly in the cytoplasm (Fig. 5AI, AII, $\mathrm{CI}, \mathrm{CII})$. In the explants exposed to FGF and insulin for $24 \mathrm{~h}$, nuclear LEDGF was reduced (Fig. 5AIII, CIII). In explants exposed to FGF and insulin for $48 \mathrm{~h}$ the cytoplasm was weakly immunopositive for LEDGF, but the nuclei were no longer LEDGF immunopositive (Fig. 5AIV, CIV). In contrast, p57 ${ }^{\mathrm{kip} 2}$, a marker for lens fiber differentiation (Zohang et al. 1997; Lovicu and McAvoy 1999), progressively and dramatically increased during the culture period $(0-48 \mathrm{~h}$; see Fig. 5DI-IV). In addition, $5^{\text {kip2 } 2}$ was weakly immunopositive at 0 or $6 \mathrm{~h}$ (Fig. 5DI, DII) and strongly immunopositive in cells exposed for 24 and $48 \mathrm{~h}$ with FGF-2 and insulin (Fig. 5DIII, DIV). Thus we found that more differentiated LECs in which $\mathrm{p} 57^{\mathrm{kip} 2}$ was immunopositive did not demonstrate nuclear LEDGF immunostaining. In control sections stained with the GST-LEDGF-neutralized $\mathrm{Ab}$ preparation, LEDGF immunostaining was absent (Fig. 5B, D). Taken together, our data from immunohistochemical staining of tissue cross-sections, LEC flat 
Fig. 6A-E In situ hybridization of LEDGF mRNA in the central LECs and bow region with biotinylated probes (blue color). In the antisense images there are positive signals overlying the anterior epithelial cells (A) and in the equatorial bow region (C), the positive signals overlying the nuclei and the cytoplasm of presumptive germinative epithelial cells (C). Inset of box indicates that the germinative zones (D) showed strong immunostaining in and/ or around the nucleus. In the cells hybridized with sense probe, there were no apparent signals overlying the nuclei or cytoplasm in central (B) or equatorial LECs (E). A-E Bar $50 \mu \mathrm{m}$, magnification $\times 100$

\section{Anterior LEC}
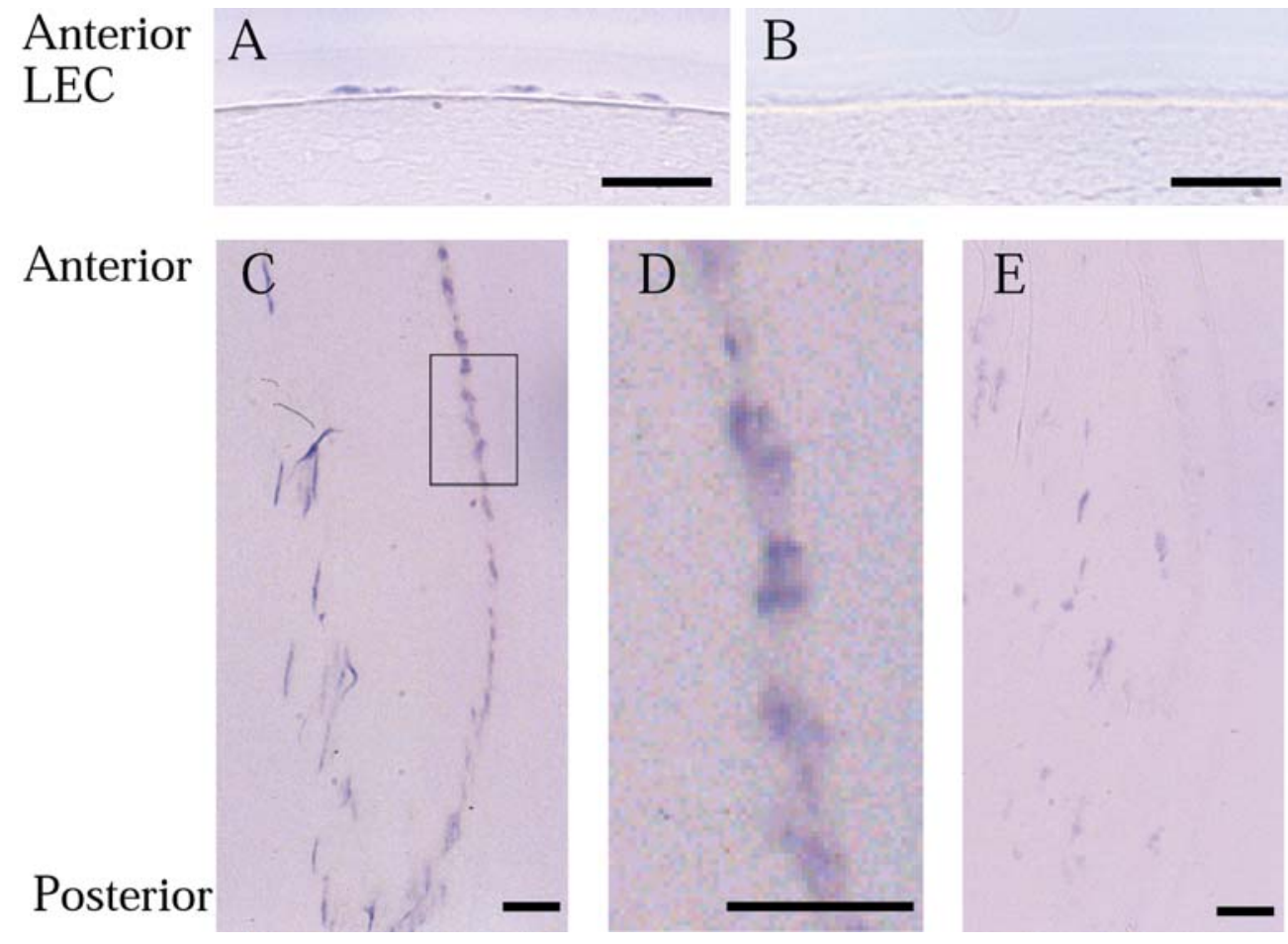

\section{Epithelium}
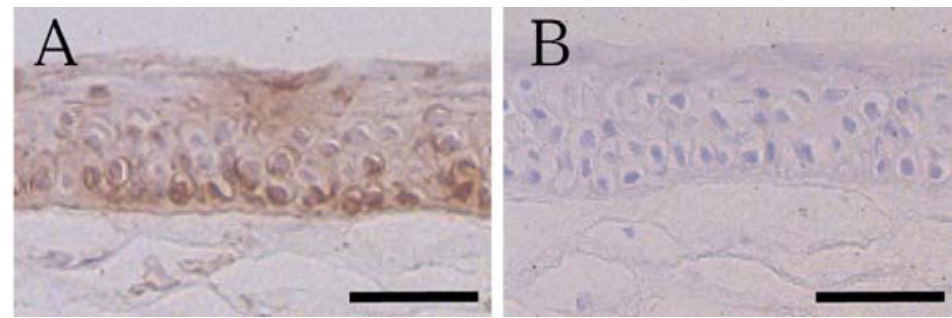

Fig. 7A, B Immunohistochemical staining of LEDGF and counterstaining in the 3-week-old rat cornea. $\mathbf{A}$ The nuclei in the basal layers of the corneal epithelium are strongly stained; the nuclei in the superficial epithelial cells are lightly stained. B Note the

preparations, lentoid bodies, and explanted LECs suggest that the process of differentiation of LECs is associated with progressive diminution of nuclear LEDGF.

\section{Localization of LEDGF mRNA in rat lens}

Next, we were keen to know LEDGF mRNA expression in the differentiating LECs. We found that LEDGF mRNA expression was decreased in the differentiating cells (Fig. 6). The hybridization of LEDGF mRNA to the LEDGF antisense probe is evident in the purple staining of the epithelial cells (Fig. 6A, C, D). In control sections (Fig. 6B, E) the hybridization of the LEDGF mRNA to the sense probe was not above the background staining. The LEDGF mRNA was present around the nucleus and the cytoplasm of epithelial cells, but it was less evident in the fiber cells (Fig. 6C). Thus, LEDGF mRNA expression also decreased in the differentiating cells. absence of specific staining in the negative control section of cornea that had been stained with the GST-LEDGF-neutralized LEDGF Ab. A, B Bar $80 \mu \mathrm{m}$, magnification $\times 100$

In rat corneal epithelium, LEDGF localized in nuclei of basal cells, but not in nuclei of superficial cells

Since corneal epithelial cells are continually renewed, basal cells divide and superficial cells shed off, we were interested to monitor LEDGF distribution in these cells or tissue. We immunolocalized LEDGF and immunohistological localization of LEDGF protein in the 3-week-old rat cornea is presented in Fig. 7. Immunostaining of LEDGF was much more intense in the nuclei than in the cytoplasm of the suprabasal and basal epithelial cells (Fig. 7A). There was a decrease in the intensity of nuclear LEDGF immunostaining between the basal and superficial layers of the epithelium. Corneal stromal and endothelial cells were weakly immunopositive for LEDGF, but it was not clear if the staining was greater in the nuclei than in the cytoplasm. In control sections stained with the GST-LEDGF-neutralized Ab preparation, LEDGF immunostaining was markedly reduced (Fig. 7B). 


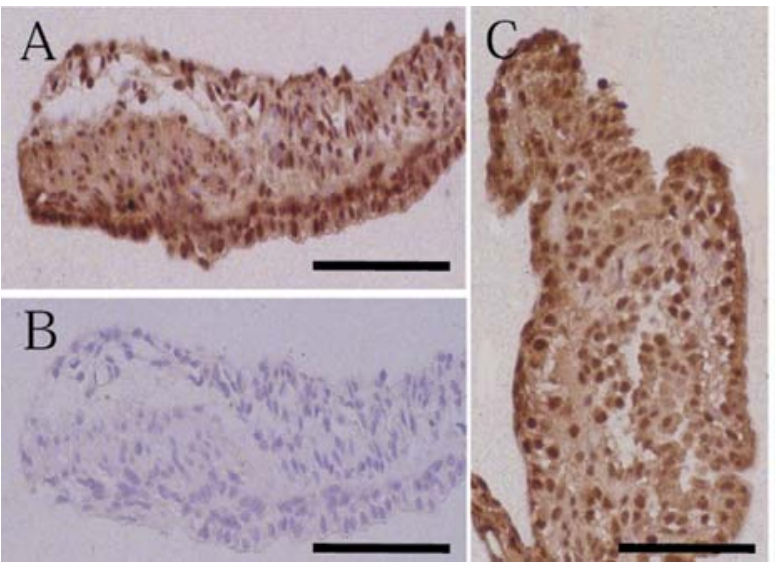

Fig. 8A-F Immunohistochemical staining of LEDGF and counterstaining in the 3-week-old rat iris $(\mathbf{A}, \mathbf{B})$, ciliary body $(\mathbf{C}, \mathbf{D})$, and retina $(\mathbf{E}, \mathbf{F})$. Positive staining for LEDGF in the nuclei of iris $(\mathbf{A})$, ciliary body $(\mathbf{C})$, and retina $(\mathbf{E})$ were observed (brown color). In

There was no difference in the pattern of LEDGF immunostaining in the cornea of 7-week-old rat (data not shown).

Localization of LEDGF in iris, ciliary body, and retinal cells

In the iris (Fig. 8A, B) and ciliary body (Fig. 8C, D), intense immunostaining was evident in the nuclei of epithelial and stromal cells; cytoplasmic staining was less intense. However, cytoplasmic staining in the cells just anterior to iris pigment epithelium was stained somewhat darker than in the epithelial cells, but there was more cytoplasmic staining on the cells just anterior to the iris pigment epithelium. In the retina (Fig. 8E, F), intense staining was evident in the inner and outer nuclear layer, and homogenous/uniform staining was evident in these inner and outer nuclear layers. Furthermore, ganglion cells were strongly stained. However, the pattern of immunolocalization was indistinguishable between the retinas of 3- and 7-week-old rats. In control sections where LEDGF neutralized anti-LEDGF $\mathrm{Ab}$ was applied, immunostaining was knocked out (Fig. 8B, D, F).

\section{Discussion}

Immunohistochemistry of LEDGF in the rat eye revealed the presence of LEDGF in the ocular tissues, lens, cornea, iris, ciliary body, and retina, with strong LEDGF immunopositivity in the nuclei and weaker immunopositivity in the cytoplasm of these cells. Interestingly, there was no detectable level of LEDGF in the nucleus of the differentiating lens fiber cells and superficial corneal epithelial cells. We further confirmed this finding and we found that LEDGF is indeed diminished significantly in the fiber cells of lentoid body, a model of LEC differentiation. Similarly, LEDGF is also lost from the nucleus of explants of LECs after 24-48 h of treatment with insulin and FGF-2 and fiber-specific protein $\mathrm{p} 57^{\mathrm{kip} 2}$ was subsequently increased in elongating LECs of the explants. Furthermore, the intensity of nuclear LEDGF immunostaining in anterior and germinative LECs was much stronger than in the nuclei of differentiating LECs. The loss of nuclear LEDGF immunopositivity began in the postequatorial region and was complete before the cells reached the equatorial bow area. The nuclei of cells in the bow area and in the superficial cortex were not immunopositive for LEDGF. Similarly in rat cornea, the intensity of nuclear LEDGF immunostaining in the basal epithelial cells was higher than in the superficial epithelial cells. The changing intensity of nuclear LEDGF immunostaining occurred in tissues undergoing TD, and this suggested to us that a more comprehensive study of LEDGF in differentiating tissues was likely to be of interest. In two of the models of TD, epithelial explants (Piatigorski 1981; Chamberlain and McAvoy 1987, 1989; Lovicu and McAvoy 1989; Chamberlain et al. 1991; Klok et al. 1998) and lentoids (Arita et al. 1988, 1990), we showed that the immunopositivity of nuclear LEDGF decreased dramatically during TD. We do not have evidence yet that the decrease in immunopositivity is due to TD or that LEDGF itself plays a primary role in TD. Interestingly, however, there is a temporal association between the apparent decrease of nuclear LEDGF and the appearance of $\mathrm{p} 57^{\mathrm{kip} 2}$ in elongating rat LEC explants exposed to FGF and insulin. P57 ${ }^{\text {kip2 }}$ is marker of differentiation in LECs (Zohang et al. 1997; Lovicu and McAvoy 1999; Carey et al. 2002) and is a member of the CIP/KIP family (Hatada and Mukai 1995; Lee et al. 1995; Matsuoka et al. 1995). Overexpression of P57 ${ }^{\mathrm{kip} 2}$ arrests cells in G1 (Hengst et al. 1994). The LECs in the equatorial region express high levels of P57 ${ }^{\text {kip2 }}$ (Zohang et al. 1997), and P57 ${ }^{\mathrm{kip} 2} \mathrm{mRNA}$ expression in adult mice is restricted to the transitional region where LECs begin 
early fiber differentiation (Lovicu and McAvoy 1999). The transformation of epithelial into fiber-like cells in the LEC explants and lentoids resembles the differentiation that occurs in the whole lens (Piatigorski 1981; Chamberlain and McAvoy 1987, 1989; Arita et al. 1988, 1990; Lovicu and McAvoy 1989; McAvoy and Chaberlain 1989; Chamberlain et al. 1991; Rampalli and Zelenka 1995; Klok et al. 1998). Lentoids also express several fiber-specific marker proteins (Arita et al. 1988, 1990). The cytoplasmic localization of LEDGF in lentoids also supports the data that nuclear LEDGF decreases in cells undergoing TD. The loss of nuclear LEDGF immunopositivity that accompanies the process of TD in LECs is similar to that which occurs in corneal epithelium (Kubo et al. 2002), where the immunopositivity of nuclear LEDGF decreases as the suprabasal cell differentiates into a superficial squamous cell. These results suggest that the loss of LEDGF from the cell nucleus might be one of the important events during the progression of lens cell fiber differentiation, and need to be investigated in detail. However, lens fiber cell differentiation represents an ideal system in dissecting the role of LEDGF, a survival factor. Moreover, it has been suggested that this could simply be due to triggering events, such as drop in cytoplasmic $\mathrm{pH}$, a fall in oxygen tension, or build up of metabolic waste occurring at the center of the lens. These events could lead to the subsequent disintegration of cell organelles and would then lead to the initiation of the denucleation and degradation of proteins due to release of proteases. Furthermore, it has been also suggested that expression of proapoptotic cytokines such as members of the tumor necrosis factor group may initiate lens fiber cell organelles loss (Wride and Sanders 1998). LEDGF is a transcription factor and induced cell survival (Fatma et al. 2000, 2001; Singh et al. 2001a), and loss or degradation of this protein by proteases may be one of the prime events toward the differentiation of cells (Wu et al. 2002). Raff's group has shown that the general caspase inhibitor (Z-VAD-FMK) is able to abolish nuclear degeneration and could protect certain proteins in an in vitro model of rat lens fiber differentiation (Ishizaki et al. 1998).

Recently, it has been observed that the process of LECs differentiating into fiber cells has biochemical parallels with apoptosis (Wride 2000). Unlike the situation in apoptosis, the lens fiber cells that lose their organelles persist throughout the life time of the organism, suggesting that there may be some important biochemical differences between the way in which apoptotic cells die and lens fiber cells lose their organelles. In fact, it has been suggested that lens fiber cell organelle loss can be considered to be a kind of attenuated apoptosis (Dahm 1999), perhaps in which the nuclear changes are biochemically dissociated from the normal changes of apoptosis such as the cytoskeleton remains intact during organelle loss in lens fibers, while in apoptosis proper it is degraded (Bassnett and Beebe 1992; Dahm et al. 1998).

In our earlier studies we have shown that in the presence of LEDGF cells survive but they die in its absence (Singh et al. 1999, 2000a; Nakamura et al. 2000). LEDGF exerts its survival function by binding to heat shock elements (nGAAn) and stress response elements (A/TGGGGA/T) and activates the expression of stressrelated proteins including Hsps in LECs (Singh et al. 2001a). Recently, several independent groups have identified that Hsp27, alpha B-crystallin, and Hsp70 exert direct, but negative influence on apoptosis signaling, and hence protect the cell (Beere et al. 2000; Bruey et al. 2000; Saleh et al. 2000). We believe that LEDGF may protect the cells via similar a mechanism as Hsps have been shown to be anti-apoptotic and LEDGF is a transactivator of Hsps (Fatma et al. 2001; Singh et al. 2001a), although further detailed investigation is required to establish the mechanism lying behind the interaction(s) and systemic regulation and expression of LEDGF and Hsps, and inhibition of caspases during lens cell fiber differentiation. However, it has been found that indeed caspase-3 triggered apoptosis in okadaic acid-induced lens epithelial cell and alpha B-crystallin could inhibit it (Li et al. 2001)

Furthermore, in the crystalline lens we found moderately intense immunostaining of LEDGF in the cytoplasm of epithelial cells and intense immunostaining of the cytoplasm in nucleated fiber cells at the equator. A most intriguing observation is that the cytoplasm of superficial cortical fibers in the anterior half of the lens is much less immunopositive for LEDGF than the cytoplasm of the superficial cortical fibers in the posterior half of the lens. It appears that there is an intracellular gradient (within the cytoplasm of superficial fibers) of LEDGF immunopositivity. Where fibers contact the capsule (posteriorly) LEDGF immunopositivity is intense, and where fibers are separated from the capsule by epithelial cells, LEDGF immunopositivity is low. Posterior fibers separated from the posterior capsule by other fibers are LEDGF immunonegative. This region of LEDGF immunonegativity corresponds roughly to the zone in which all, or almost all, cytoplasmic organelles are absent in the lens fiber. The significance, if any, of this observation is not yet clear, but it is interesting to consider that cytoplasmic LEDGF levels might also influence the later stages of TD. Furthermore, we found the intense nuclear staining of LEDGF in retina, ciliary body, and iris, where the cells are well-differentiated. We have reported that LEDGF promotes cellular survival of retinal photoreceptor cells and human retinal pigment epithelium (Nakamura et al. 2000; Machida et al. 2001; Matsui et al. 2001). However, the localization pattern of LEDGF indicates that it may have different functions in different cells, that may depend on the stage and type of cells and its intracellular distribution.

The in situ hybridization experiments with sections of rat lenses have shown that the mRNA for LEDGF is present, even abundant, in the epithelial cells of the pregerminative, germinative, and the most superficial equatorial zones. A reduction in the amount of LEDGF mRNA begins and progresses rapidly in more deeply located fibers, and in the deepest cortical fibers there is no 
LEDGF mRNA. The presence of LEDGF mRNA in the superficial cortex confirms that LEDGF transcription is active there. This is intriguing for it suggests that there are extranuclear functions for LEDGF in the cell. The transcription and pre-mRNA splicing roles would be effected in the nucleus. LEDGF localizes in the cytosol of cultured LECs at $15^{\circ} \mathrm{C}$ and $28^{\circ} \mathrm{C}$ (Singh et al. 1999). However, we do not yet understand what might be the normal extranuclear functional roles of LEDGF. LEDGF localizes in nucleus (Singh et al. 2001b), and furthermore, phosphorylation of LEDGF might also modify its intracellular distribution. LEDGF is a phosphoprotein and phosphorylation alters the intracellular localization of many proteins. One example is basonuclin, a zinc finger protein (Iuchi and Green 1997) and transcription factor (Tseng et al. 1999). We might expect the subcellular distribution of LEDGF to change as a function of the phosphorylation status of LEDGF. We have recently begun a series of experiments focusing on how phosphorylation alters the localization and functional properties of LEDGF.

So far three known transcription factors are reported to determine the fate of LECs: (1) loss of LEDGF in the LECs using antisense LEDGF cDNA-induced cell death (unpublished data), (2) loss of retinoblastoma susceptibility gene, a negative regulator of the cell proliferation, also reported to induce cell death (Tan et al. 1997), and (3) Creb-2, an evolutionary conserved member of the CREB/ATF family of basic-leucin zipper transcription factor, is reported to be an important regulator of mammalian lens development (Hettmann et al. 2000).

Further detailed studies will be required to elucidate the mechanism involved in the expression and localization of this physiologically important gene. In summary, however, we established a temporal relationship between the loss of LEDGF from nucleus and fiber cell differentiation. In addition, our findings add weight to the hypothesis that LEDGF is one of the factors that may play a regulatory role in determining the fate of cells. We believe our continuing studies of LEDGF on the functional role of cytoplasmic or nuclear LEDGF within the cell, the fate of LEDGF during differentiation and apoptosis, and the effect of proteases on LEDGF, will provide a better understanding of the roles of LEDGF in cell survival and differentiation.

Acknowledgements The work was supported by NIH grants EY12015, EY10824, EY13394, and EY07003, and the Massachusetts Lions Eye Research Fund.

\section{References}

Arita T, Lin LR, Reddy VN (1988) Differentiation of human lens epithelial cells in tissue culture. Exp Eye Res 47:905-910

Arita T, Lin LR, Susan SR, Reddy VN (1990) Enhancement of differentiation of human lens epithelium in tissue culture by changes in cell-substrate adhesion. Invest Ophthalmol Vis Sci 31:2395-2404
Balaram M, Tung WH, Kuszak J, Ayaki M, Shinohara T, Chylack LT Jr (2000) Non-contact specular microscopy of human lens epithelium. Invest Ophthalmol Vis Sci 41:474-481

Bassnett S, Beebe DC (1992) Coincident loss of mitochondria and nuclei during lens fiber cell differentiation. Dev Dyn 194:85-93

Beere HM, Wolf BB, Cain K, Mosser DD, Mahbboudi A, Kuwana, T, Railor P, Moromoto RI, Cohen GM, Green DR (2000) Heat shock protein 70 inhibits apoptosis by preventing the recruitment of procaspase-9 to the Apaf-1 apoptosome. Nat Cell Biol $2: 469-475$

Bradford MM (1976) A rapid sensitive method for the quantification of microgram quantities of protein utilizing the principle of protein dye binding. Anal Biochem 72:248-254

Bruey JM, Ducasse C, Bonniaud P, Ravagnan L, Susin SA, SiazLatoud C, Gurbuxani S, Arrigo AP, Kroemer G, Solary E, Garrido C (2000) Hsp-27 negatively regulates cell death by interacting with cytochrome c. Nat Cell Biol 2:645-652

Carey RG, Li B, DiCicco-Bloom E (2002) Pituitary adenylate cyclase activating polypeptide anti-mitogenic signaling in cerebral cortical progenitors is regulated by p57Kip2-dependent CDK2 activity. J Neurosci 22:1583-1591

Chamberlain CG, McAvoy JW (1987) Evidence that fibroblast growth factor promotes lens fiber differentiation. Curr Eye Res 6:1165-1168

Chamberlain CG, McAvoy JW (1989) Induction of lens fiber differentiation by acidic and basic fibroblast growth factor (FGF). Growth Factors 1:125-134

Chamberlain CG, McAvoy JW, Richardson NA (1991) The effects of insulin and basic fibroblast growth factor on fiber differentiation in rat lens epithelial explants. Growth Factors 4:183-188

Dahm R (1999) Lens fiber cell differentiation: a link with apoptosis. Ophthalmic Res 31:163-183

Dahm R, Gribbon C, Quinlan RA, Prescott AR (1998) Changes in nucleolar and coiled body compartments precede lamina and chromatin reorganization during fiber cell denucleation in the bovine lens. Eur J Cell Biol 75:237-246

Fatma N, Singh DP, Shinohara T, Chylack LT Jr (2000) Heparin's roles in stabilizing, potentiating and transporting LEDGF into the nucleus. Invest Ophthalmol Vis Sci 41:2648-2657

Fatma N, Singh DP, Shinohara T, Chylack LT Jr (2001) Transcriptional regulation of the antioxidant protein 2 gene, a thiol-specific antioxidant, by lens epithelium-derived growth factor to protect cells from oxidative stress. J Biol Chem 276:48899-48907

Ge H, Si Y, Roeder RG (1998a) Isolation of cDNAs encoding novel transcription co-activators p52 and p75 reveals an alternate regulatory mechanism of transcriptional activation. EMBO J 17:6723-6729

Ge H, Si Y, Wolffe AP (1998b) A novel transcriptional coactivator, p52, functionally interacts with the essential splicing factor ASF/SF2. Mol Cell 2:751-759

Hatada I, Mukai T (1995) Genomic imprinting of $\mathrm{p} 57^{\mathrm{kip} 2}$, a cyclindependent kinase inhibitor, in mouse. Nat Genet 11:204-206

Hengst L, Duvic V, Slingerland JM, Lees E, Red SI (1994) A cell cycle-regulated inhibitor of cyclin-dependent kinases. Proc Natl Acad Sci U S A 91:5291-5295

Hettmann T, Barton K, Leiden JM (2000) Microphthalmia due to p53-mediated apoptosis of anterior lens epithelial cells in mice lacking the CREB-2 transcription factor. Dev Biol 222:110 123

Ibaraki N, Lin LR, Reddy VN (1995) Effects of growth factors on proliferation and differentiation in human lens epithelial cells in early subculture. Invest Ophthalmol Vis Sci 36:2304-2312

Ishizaki Y, Voyvodic JT, BurneJF, Raff MC (1993) Control of lens epithelial cell survival. J Cell Biol 121:899-908

Ishizaki Y, Jacobson MD, Raff MC (1998) A role of caspases in lens fiber differentiation. J Cell Biol 140:153-158

Iuchi S, Green H (1997) Nuclear localization of basonuclin in human keratinocytes and the role of phosphorylation. Proc Natl Acad Sci U S A 94:7948-7953

Klok E, Lubsen NH, Chamberlain CG, McAvoy JW (1998) Induction and maintenance of differentiation of rat lens 
epithelium by FGF2, insulin and IGF-1. Exp Eye Res 67:425431

Kubo E, Fatma N, Shinohara T, Chylack LT Jr, Akagi Y, Singh DP (2002) Transactivation of involucrin, a marker of differentiation in keratinocytes, by lens epithelium derived growth factor (LEDGF). J Mol Biol 320:1053-1063

Lee MH, Reynisdottir I, Massague J (1995) Cloning of p57kip2, a cyclin-dependent kinase inhibitor with unique domain structure and tissue distribution. Genes Dev 9:639-649

Li DW, Xiang H, Mao YW, Wang J, Fass U, Zhang XY, Xu C (2001) Caspase-3 is actively involved in okadaic acid-induced lens epithelial cell apoptosis. Exp Cell Res 266:279-291

Lovicu FJ, McAvoy JW (1989) Structural analysis of lens epithelial explants induced to differentiate into fibers by fibroblast growth factor (FGF). Exp Eye Res 49:479-494

Lovicu FJ, McAvoy JW (1999) Spatial and temporal expression of p57 ${ }^{\text {kip2 }}$ during murine lens development Mech Dev 86:165-169

Machida S, Chaudhary P, Shinohara T, Singh DP, Reddy VN, Chylack LT Jr, Sieving PA, Bush RA (2001) Lens epitheliumderived growth factor promotes photoreceptor survival in lightdamaged and RCS rats. Invest Ophthalmol Vis Sci 42:10871095

Matsui H, Lin LR, Singh DP, Shinohara T, Reddy VN (2001) Lens epithelium-derived growth factor: increased survival and decreased DNA breakage of human RPE cells induced by oxidative stress. Invest Ophthalmol Vis Sci 42:2935-2941

Matsuoka S, Edwards MC, Bai C, Parker S, Zhang P, Baldini A, Harper JW, Elledge SJ (1995) p57 kip2, a structurally distinct member of the p21 kip1 $\mathrm{Cdk}$ inhibitor family, is a candidate tumor suppressor gene. Genes Dev 9:650-662

McAvoy JW, Chamberlain CG (1989) Fibroblast growth factor (FGF) induced different responses in lens epithelial cells depending on its concentration. Development 107:221-228

Nakamura M, Singh DP, Kubo E, Chylack LT Jr, Shinohara T (2000) LEDGF: survival of embryonic chick retinal photoreceptor cells. Invest Ophthalmol Vis Sci 41:1168-1175

Piatigorsky J (1981) Lens differentiation in vertebrates: a review of cellular and molecular features. Differentiation 19:134-153

Raff MC (1992) Social controls on cell survival and cell death. Nature 356:397-400

Rampalli AM, Zelenka PS (1995) Insulin regulates expression of cfos and c-jun and suppresses apoptosis of lens epithelial cells. Cell Growth Differ 6:945-953

Renaud F, Oliver L, Desset, Tassin J, Romquin N, Courtois Y, Laurent M (1994) Up-regulation of FGF-1 expression in quiescent cells is related to cell survival. J Cell Physiol 158:435-443

Saleh A, Srinivasula SM, Balki L, Robbins PD, Alnemri ES (2000) Negative regulation of Apaf-1 apoptosome by Hsp70. Nat Cell Biol 2:476-483
Sharma P, Singh DP, Fatma N, Chylack LT Jr, Shinohara T (2000) Activation of LEDGF gene by thermal- and oxidative-stresses. Biochem Biophys Res Commun 276:1320-1324

Singh DP, Ohguro N, Chylack LT Jr, Shinohara T (1999) Lens epithelium-derived growth factor: increased resistance to thermal and oxidative stresses. Invest Ophthalmol Vis Sci 40:1444-1451

Singh DP, Ohguro N, Kikuchi T, Sueno T, Reddy VN, Yuge K, Ko V, Chylack LT Jr, Shinohara T (2000a) Lens epitheliumderived growth factor: effects of growth and survival of lens epithelial cells, keratinocytes, and fibroblasts. Biochem Biophys Res Commun 267:373-381

Singh DP, Kimura A, Chylack LT Jr, Shinohara T (2000b) Lens epithelium-derived growth factor (LEDGF/p75) and p52 are derived from a single gene by alternative splicing. Gene 242:265-273

Singh DP, Fatma N, Kimura A, Chylack LT Jr, Shinohara T (2001a) LEDGF binds to heat shock and stress-related element to activate the expression of stress-related genes. Biochem Biophys Res Commun 283:943-955

Singh DP, Fatma N, Chylack LT Jr, Shinohara T (2001b) Distinct nuclear localization signal and DNA-binding domains of LEDGF. Invest Ophthalmol Vis Sci (suppl) 42:2909

Stolen CM, Jackson MW, Griep AE (1997) Overexpression of FGF-2 modulates fiber cell differentiation and survival in the mouse lens. Development 124:4009-4017

Tan X, Martin SJ, Green DR, Wang JY (1997) Degradation of retinoblastoma protein in tumor necrosis factor- and CD95induced cell death. J Biol Chem 212:9613-9616

Tseng H, Matsuzaki K, Lavker RM (1999) Basonuclin in murine corneal and lens epithelia correlates with cellular maturation and proliferative ability. Differentiation 65:221-227

Wride MA (2000) Apoptosis as seen through the lens. Apoptosis 5:203-209

Wride MA, Sanders EJ (1998) Nuclear degeneration in the developing lens and its regulation by TNF alpha. Exp Eye Res 66:371-383

Wu X, Daniels T, Molinaro C, Lilly MB, Casiano CA (2002) Caspase cleavage of the nuclear autoantigens LEDGF/p75 abrogates its pro-survival function: implication for autoimmunity in atopic disorders. Cell Death Differ 9:915-925

Zohang P, Liégeois NJ, Wong C, Finegold M, Hon H, Thompson JC, Silverman A, Harper JW, DePinho RA, Elledge SJ (1997) Altered cell differentiation and proliferation in mice lacking p57 $7^{\text {kip2 }}$ indicates a role in Beckwith-Wiedermann syndrome. Nature 387:151-158 